\title{
Tipos de conocimiento y preferencias para la resolución de problemas
}

${ }^{(1)}$ Adolfo Oswaldo Acevedo Borrego

(2) Carolina Linares Barrantes

(3) Orestes Cachay Boza

\section{RESUMEN}

El conocimiento, desde la perspectiva de los griegos, es de cuatro tipos: empeiría, tékhne, epistéme y noús. Estos tipos presentan diferentes características, según se orienten a la dimensión del origen (hechos o conceptos), 0 a la dimensión de la finalidad (contemplación 0 dominio).

Sobre la base de estas dimensiones se plantea una herramienta para percibir, desarrollar y consolidar las fortalezas personales, derivadas de la predisposición del propio conocimiento. Esta herramienta permite delinear el potencial productivo personal, bajo la perspectiva que el conocimiento adquirido determina la predisposición y el desarrollo de su capacidad para tomar decisiones y resolver problemas.

En la muestra estudiada, se observa que los profesionales tienen diferente predisposición hacia las dimensiones del conocimiento, lo que conlleva un diferente perfi personal para la resolución de problemas. Según el perfil predominante, los profesionales están más orientados al análisis y estudio, y menos a resolver problemas y la orientación al dominio es baja. En general, el presente estudio brinda elementos de juicio para ampliar la perspectiva de desarrollo y aprendizaje profesional.

Palabras clave: Conocimiento, dimensiones de conocimiento, perfil directivo, enfoques metodológicos, resolución de problemas.

TYPES OF KNOWLEDGE AND PREFERENCES FOR SOLVING PROBLEMS

\section{ABSTRACT}

The Knowledge, from the perspective of the Greeks, it has four types: Empeiria, tekhne, episteme and nous. These types have different characteristics, as they target the source dimension (facts and concepts), or the purpose dimension (contemplation or domain).

On the basis of these dimensions is presented a tool kit to collect, develop and build personal strengths, arising from the disposition of knowledge itself. This tool allows to outline the productive potential of staff, from the perspective that the knowledge acquired, determines the disposition and development of ability to make decisions and solve problems.

In the sample studied, it was noted that professionals have different susceptibility to the dimensions of knowledge, which leads to a different personal profile for the resolution of problems. According to the predominant profile, professionals are more focused on the analysis and study, and less problem solving and mastery orientation is low. In general, this study provides evidence to broaden the perspective of development and professional learning.

Keywords: Knowledge, knowledge dimensions, profile management, methodological approaches, troubleshooting.

\section{INTRODUCCIÓN}

Las profesiones orientadas a la aplicación de la ciencia para la solución de problemas de empresa, como son la Investigación de operaciones, la Ingeniería Industrial y la Ingeniería de Sistemas, se encargan del diseño, planeación, implantación y manejo de sistemas de actividad humana o SAH. Se enfocan en la creación y gestión de sistemas de producción conformados por personas y cosas (máquinas, materiales, ambiente), empleando herramientas técnicas orientadas a elevar la productividad, mejorar la eficiencia, lograr la mayor eficacia y resolver problemas empresariales complejos.

Para resolver los problemas de los sistemas productivos, se requiere el mejor ajuste entre el talento del ingeniero, el caso a enfrentar y el tipo de conocimiento adecuado. El presente trabajo se refiere al diseño de un modelo conceptual de decisiones basado en la predisposición a los diferentes tipos de conocimiento. Se intenta identificar la orientación que el ingeniero posee, definido como las características o aptitudes que puede desarrollar plenamente si las circunstancias generan las condiciones adecuadas para acceder al tipo de conocimiento adecuado que permita el despliegue de su potencial de toma de decisiones.

\section{ANTECEDENTES}

Los primeros antecedentes se encuentran en los filosófos griegos. Aristóteles afirma que el acceso de la verdad viene por el conocimiento del mundo sensible y define los tipos de conocimiento teórico y práctico. Por otro lado, Platón considera que el mundo sensible es un reflejo de las ideas, diferenciando opinión que deviene de la imaginación y las creencias, y conocimiento que deriva del entendimiento y la inteligencia. Hume define que el conocimiento deviene de las impresiones o las experiencias sensibles, donde las ideas son simples asociaciones de estas experiencias.

1 Magíster en Administración, Ingeniero Industrial, Economista. Profesor en la Facultad de Ingeniería Industrial, Departamento de Producción y Gestión Industrial de la UNMSM. E-mail: aacevedo@speedy.com.pe

2 Magíster en Dirección de Empresas por la Universidad de Piura, Ingeniero Industrial, estudios de Derecho. Profesora en la Facultad de Ingeniería de Sistemas e Informática UNMSM. E-mail: klinares@speedy.com.pe

3 Doctor en Ingeniería Industrial, Ingeniero Industrial. Profesor en la Facultad de Ingeniería Industrial, Departamento de Producción y Gestión Industrial de la UNMSM. Email: orestescachay@yahoo.es 
Los antecedentes académicos se encuentran en la teoría $X-Y$ sobre la actitudes de los trabajadores (McGregor, 1970) y los primigenios conceptos de eficacia directiva enunciados por Peter Drucker. Los nuevos estudios enfatizan la naturaleza cambiante del trabajo y el sentido de la incertidumbre en el futuro, derivada de la paradoja de la certeza de los pensamientos opuestos dialécticos de continuidad y cambio (Handy, 1997), es relevante el trabajo sobre el modelo de aprendizaje y solución de problemas por experiencias que plantea cuatro estilos de aprendizaje basado en las aptitudes de los directivos, son los estilos divergente, asimilador, convergente y acomodador (Kolb, Rubin \& Maclntyre, 1977).

La nueva corriente de las organizaciones inteligentes define que el don o talento para el desarrollo de las cinco disciplinas de aprendizaje es una práctica que exige estudio y concentración (Senge, 1994). EI Instituto de Ingenieros Industriales de USA amplía el aspecto humano del modelo sociotécnico, planteando que las organizaciones deben considerar, en forma equilibrada y simultánea, el diseño técnico y el comportamiento humano, este análisis relaciona la estructura de la tarea con las necesidades y las expectativas del individuo, concluyendo que la gestión debe considerar el sentido de la tarea y el significado del hombre para sí mismo, dentro de la pertinencia de los factores ambientales (Vaill, 1967).

En el .ámbito de la UNMSM, se han realizado estudios enfocados en la dimensión humana de los sistemas (persona-tarea). García ha estudiado la creatividad, destrezas, motivaciones y satisfacción de los alumnos de ingeniería industrial. El estudio de Cevallos considera los conceptos educativos para la nueva universidad del siglo XXI. El estudio de Loli Pineda analiza los factores de compromiso organizacional en relación con las variables de satisfacción y grado de compromiso afectivo/conveniencia de los trabajadores universitarios con su institución. El estudio de García\&Vergiu\&Párraga\&Santos se refiere a la medición del capital intelectual empresarial, definiéndolo como los activos intangibles materializados en destrezas, conocimiento técnico, sistemas de información, diseños, marcas y relaciones con proveedores, intermediarios y clientes.

En la actualidad se afirma que la aptitud natural de la inteligencia para entender la complejidad del mundo, se desarrolla mediante la ubicación del conocimiento o informaciones en un contexto y un conjunto (Morín, 1999). Así, el presente estudio se enmarca dentro de las teorías de la escuela de sistemas, descrita por Blair y Whitson, específicamen- te en el diseño de sistemas de actividad humana, en su componente persona.

\section{DIMENSIONES DEL CONOCIMIENTO}

\section{El talento de resolución de problemas}

Toda persona posee características o aptitudes que le permiten identificar situaciones problemáticas y resolverlas, dentro de las organizaciones. El talento de resolución de problemas se puede desarrollar, en mayor o menor medida, a partir de ciertas condiciones de entorno, de adquisición de conocimiento, de actitud personal y fuerzas circunstanciales que confluyen para desplegar dicho talento. Dentro de este modelo, el proceso clave es la adquisición de conocimiento.

El talento para la resolución de problemas es la aptitud o el potencial de las personas, para desarrollar un conjunto de habilidades o competencias para la mejora de productividad y eficiencia empresarial. También se define que el talento es un conjunto de aptitudes y destrezas por las que una persona sobresale y obtiene resultados calificados de notables o exitosos. El talento, mientras no se despliegue, se considera como potencial.

\section{Los tipos de conocimiento}

Aristóteles, en su obra Metafísica, afirma que "Todos los hombres tienden por naturaleza a saber", señal de ello es el gusto por las sensaciones, distingue el uso por su utilidad para hacer algo (Marías, 2007).

Se definen diversos tipos de conocimiento: Primero, el ínfimo saber cuya señal es el gusto por las sensaciones, lo poseen el hombre y los animales, el aprendizaje se deriva de la permanencia del recuerdo en la memoria. Existe el saber por la experiencia o empeiría, es un modo superior de saber, es la familiaridad de las cosas de forma inmediata y concreta, éste no se puede enseñar. Segundo, la tékhne, el arte o técnica es el saber hacer las cosas, se define como un saber superior, el cual se puede enseñar. Tercero, la ciencia o epistéme es el saber demostrativo que organiza y sistematiza el conocimiento en diversas especialidades. Permite conocer las causas y principios primeros. Cuarto, el noús o conocimiento intuitivo que permite intuir los principios no demostrables, los que surgen espontáneamente por iluminación y que parece que no se derivan de nada. Finalmente se define el saber supremo, la sophía o verdadera sabiduría, que está compuesto por los momentos esenciales de la noús y el epistéme. Este esquema se sintetiza en 
las dos corrientes escolásticas enfrentadas (Tomás Aquinas y Agustín de Hippo), que definen que el fin del hombre es la búsqueda del saber verdadero versus [es] la búsqueda del bien verdadero (Hinnells, 1995).

\section{Dimensiones del conocimiento}

Para definir las dimensiones del conocimiento, se han de considerar dos continuos. El continuo del entendimiento donde se determina que el conocimiento llega a través de los sentidos y es comprendido a través de la razón, ambos representan opuestos dialécticos del eje $y$ del entendimiento. En el continuo de la finalidad, Aristóteles considera que el fin del conocimiento es la contemplación de la naturaleza, en contraposición, Bacon afirma que el fin es el dominio de la naturaleza, son los opuestos dialécticos del eje $x$ de la finalidad.

Ambos continuos se visualizan como coordenadas cartesianas (Gráfico 1). Dentro del continuo del entendimiento, se tienen los opuestos realidadideas, en un extremo, los hechos de la realidad son percibidos por los sentidos como fenómenos de la naturaleza, en el otro extremo, lo percibido por los sentidos llega al entendimiento por las ideas y el pensamiento, a través de los conceptos y paradigmas se comprende y se acepta el conocimiento verdadero. Dentro del continuo de la finalidad, se tienen los opuestos contemplación-dominio, en un extremo, Aristóteles sostiene la perspectiva de la contemplación de la naturaleza a través de la ciencia y la filosofía, en el otro extremo, Bacon tiene la perspectiva del dominio de la naturaleza a través del progreso técnico y la tecnología.

Las dimensiones del conocimiento son cuatro: hechos de la realidad, fenómenos o problema, paradigmas y conceptos, contemplación u observación y el dominio de la naturaleza. Estas dimensiones se complementan con la definición aristotélica de los cuatro tipos de conocimiento demostrable: empírico, científico, técnico e intuitivo. El modelo concuerda con el enfoque de las revoluciones científicas (Kuhn, 1971), en el eje y define las dimensiones del mundo real y mundo percibido (problemas de la realidad frente a paradigmas para entenderlos), en el eje $x$ define las dimensiones de la reducibilidad y estudio analítico del problema identificado y la toma de decisión para la solución de problemas (rompecabezas o partes frente a la resolución del problema).

Gráfico 1. Los tipos de conocimiento.

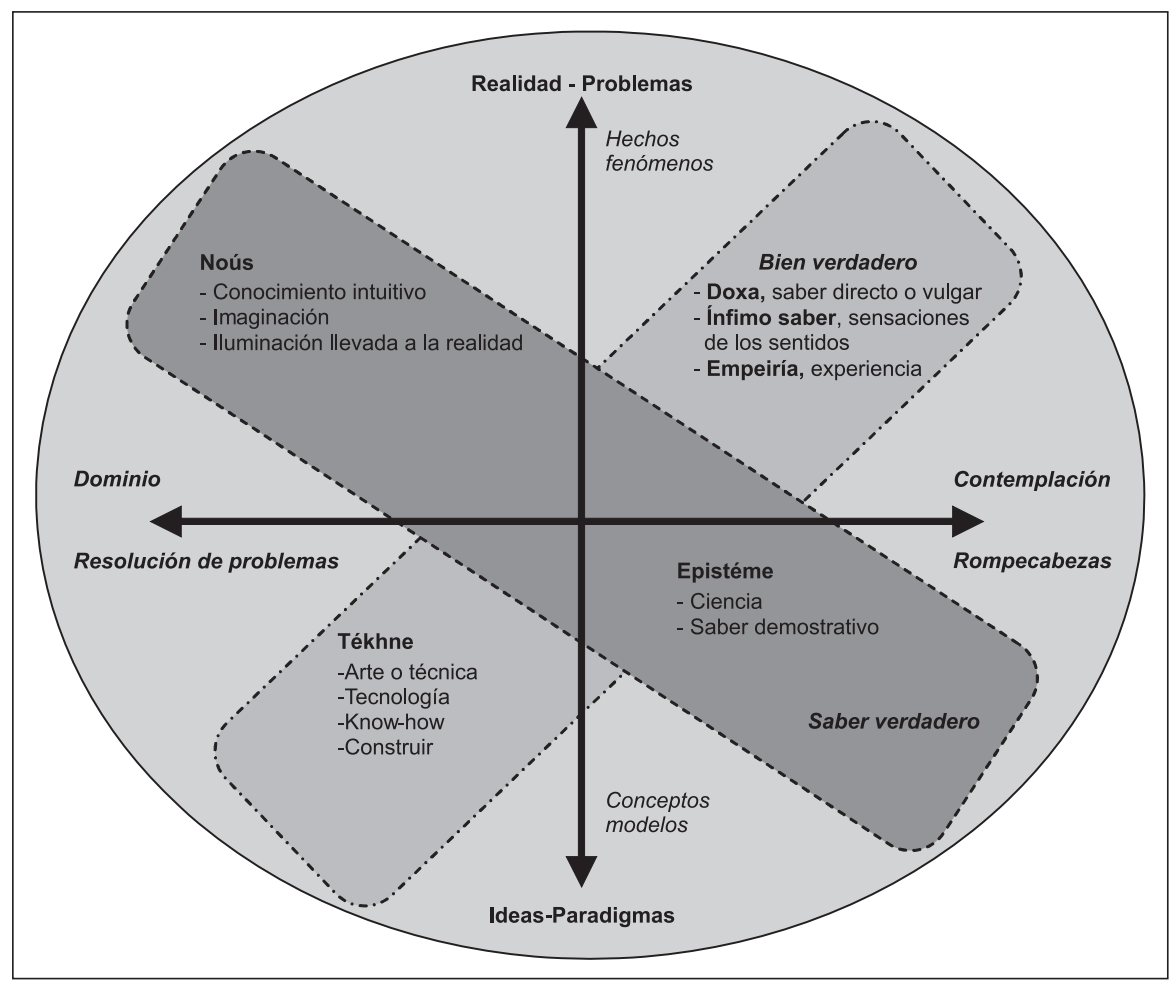

Fuente: Acevedo (2009). "Identificación del talento potencial en los alumnos de Ingeniería Industrial”. 


\section{MODELO DE PERFIL DIRECTIVO PARA LA RE- SOLUCIÓN DE PROBLEMAS}

\section{Los ejes cartesianos del modelo de conocimiento}

Este modelo se refiere a elementos de toma de decisiones y resolución de problemas en organizaciones, cuyas dimensiones son persona y circunstancia. La persona es el decisor que tiene fines, los cuales pueden ser el conocer los hechos o controlar estos hechos. La circunstancia en el entorno real, que contiene los hechos externos definidos como eventos probabilísticos que se enlaza con los fines y acciones del decisor, cuya conducta es no determinística (Gráfico 2).

La problemática empresarial (que es la naturaleza de las cosas) se percibe a través de la dimensión de los fenómenos y hechos, lo que se perciben sensorialmente y se entienden mediante los conceptos que se tienen sobre las cosas, mediante la racionalidad y el pensamiento. Es el eje y que se representa como el continuo Realidad-Razón, que contrapone los hechos a los conceptos. Este eje contiene la dimensión de los hechos y la dimensión de los conceptos.

Los fenómenos de la realidad son tomados para conocerlos, por un lado se desagregan como un rompecabezas y se analizan, con lo que se genera saber básico, por otro lado, se busca el dominio del ambiente y la resolución de los problemas, para lo cual la persona elige y genera acción. Es el eje $x$ que se representa como el continuo contemplacióndominio, que contrapone el observador estudioso frente al decisor. Este eje contiene la dimensión de la contemplación y la dimensión del dominio.

\section{Enfoques metodológicos para la resolución de problemas}

La intersección de estos ejes de coordenadas, conforma cuatro campos de actuación, los que definen la epistemología y metodología de generación y empleo del conocimiento.

El primer campo es el campo del hacer y quehacer. Relaciona los hechos de la realidad con las decisiones pragmáticas de controlar las cosas del entorno, es el enfoque utilitarista de los problemas, orientado a las mejoras concretas antes que a las teorías, enfatizando la satisfacción de las preferencias del decisor. Es el campo de las invenciones técnicas, tecnología, manejo de recursos. Es el campo del foco de problemas, pragmatismo y elección de alternativas. Este conocimiento se resume en principios, teorías y leyes organizados y sistematizados, es gestionable y transmisible.

El segundo campo es el campo de lo dado, de lo ya hecho, lo que se ha recibido de la naturaleza circundante. Combina la realidad social y económica con la observación de los fenómenos de esa realidad, es el enfoque positivista de los problemas, está orientado a la realidad concreta y enfatiza la experiencia del mundo real, es el empirismo objetivo de las decisiones. Es el campo de las sensaciones, este conocimiento se capta a través de los sentidos, se estima que se aprende pero no se puede enseñar. Se emplean metodologías de aprendizaje por experiencia.

El tercer campo relaciona los conceptos con el afán de controlar el entorno, es el enfoque constructivista de los problemas, orientado a la formulación, diseño de modelos físicos y conceptuales y teorías reflexivas aplicadas a la solución de problemas, incluyendo su verificación en los hechos del mundo real. Es un enfoque que integra los conceptos con la realidad objetiva, considera la síntesis como siguiente paso del análisis. Es el campo de la creación, las visiones para construir, crear y avanzar, es el campo de la praxis y acción emprendedora, este conocimiento es racional, e integra ideas innatas con la resolución empírica de problemas.

El cuarto campo integra la observación con los constructos del observador, es el campo del enfoque racionalista de los problemas, está orientado al estudio, descubrimiento y experimentación de las leyes de la realidad, mediante el estudio sistemático y enfoque inductivo, es el investigador, analítico y experimental. Es el campo de la abstracción y el análisis sistemático, este conocimiento se acumula, se organiza y se gestiona como saber básico sin inmediata, representa las bases teóricas cuya aplicación práctica genera el conocimiento tecnológico. 
Gráfico 2. Marco metodológico para la resolución de problemas.

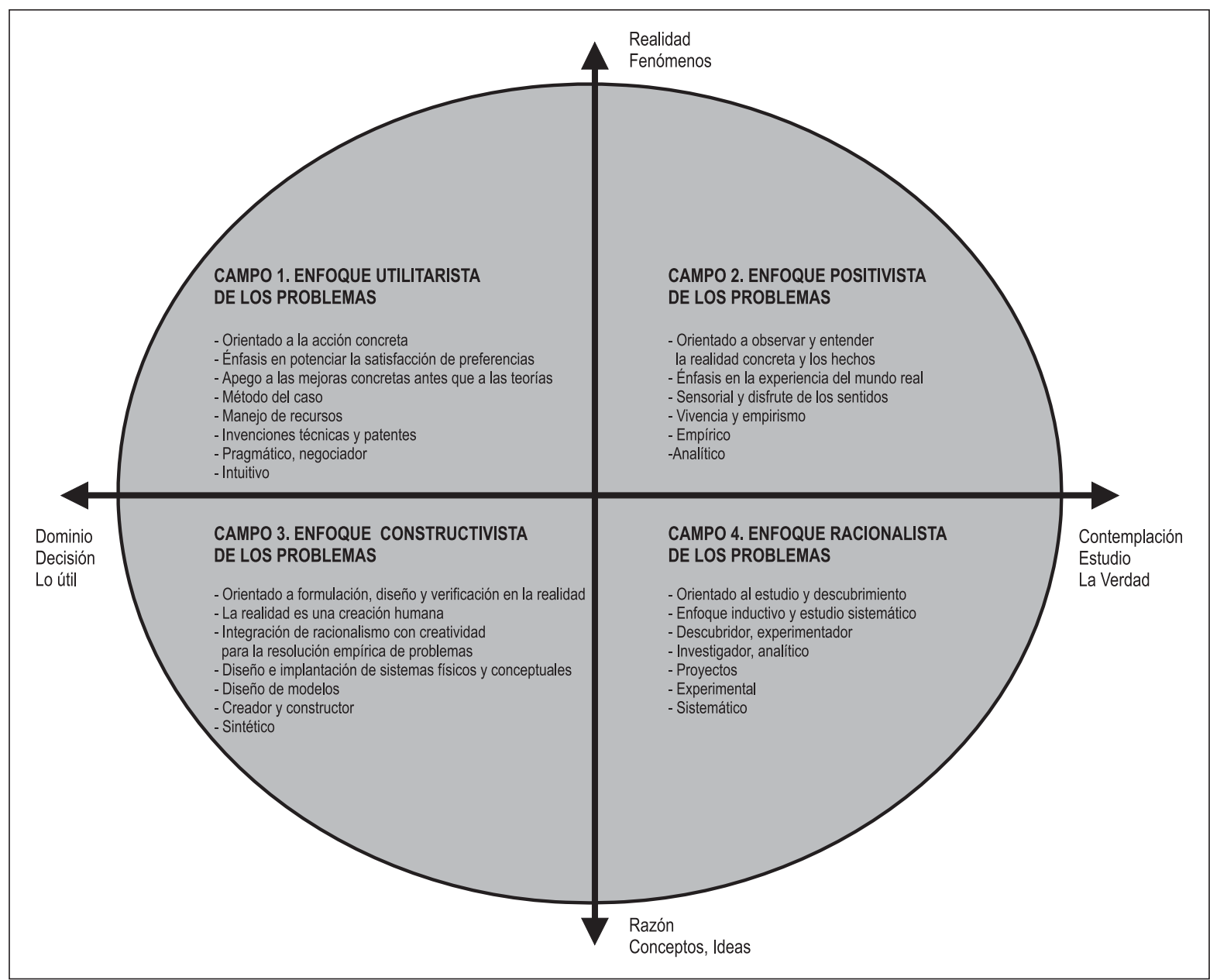

Fuente: Ibídem

\section{Los perfiles del decisor según los tipos de co-} nocimiento

La predisposición personal hacia uno u otro de estos campos, es determinante en la definición de las características del potencial de decisiones de los profesionales.

De manera a priori, se estima que los profesionales de cualquier especialidad, adquieren una predisposición a resolver los problemas, enfocándolos con una metodología específica, aprendida y aprehendida durante los años de su formación académica. En el caso de las profesiones enfocadas en el diseño y funcionamiento de sistemas industriales, posiblemente habrían de tener un mayor acercamiento hacia el campo del dominio y el utilitarismo, ya que este campo se acerca al perfil de formación de los ingenieros como solucionadores de problemas y gestionadores de recursos y personas, como se describe en la definición de los sistemas de actividad humana.

Para describir los cuatro campos de actuación del ingeniero, se ha considerado el perfil público. El campo 1 es el ámbito de gestión de hechos y manejo de recursos, enfoca temas concretos con criterio pragmático, aunque puede crear soluciones a problemas reales mediante procesos mentales no controlados a lo que se denomina lluminación o Revelación, cuyo resultado son las creaciones y los inventos y el emprendedurismo. El campo 2 es el ámbito de la acción real, las personas poseen intereses concretos de conocer cómo funcionan las cosas, más orientado al conocimiento sensible de los hechos que a la solución de la problemática, puede ser el caso del artista o literato, con enfoques amplios. El campo 3 es el ámbito de la síntesis y la 
creatividad para la solución de los problemas, son diseñadores de teorías, modelos y sistemas físicos y conceptuales, cuya validez se comprueba con su funcionamiento eficaz en la realidad. El campo 4 es el ámbito de la visión analítica, la previsión y el estudio sistematizado, deviene en pensamiento abstracto y análisis predictivo, es característico de los académicos e investigadores universitarios.

\section{PLANTEAMIENTO DE HIPÓTESIS Y DISEÑO EX- PERIMENTAL}

\section{El diseño de la investigación}

Dentro del Problema de Investigación definido como " ¿Es posible determinar si existe predisposición del ingeniero hacia una determinada dimensión? ¿De ser así, de qué manera afecta la elección del decisor?".

Para resolver la pregunta del problema de investigación, cuya hipótesis principal fue: "Los ingenieros industriales poseen un talento potencial equilibrado, el cual se puede desplegar a medida que se amplíen las posibilidades de aplicación del tipo de conocimiento adecuado"4, se diseñó un experimento que considera el efecto de las dimensiones en la elección del decisor, cuando enfrenta diferentes situaciones. Luego se diseñó un experimento completamente aleatorio donde se comparan $\mathrm{k}$ tratamientos del factor $\mathrm{D}$ (dimensiones), a este tipo de experimento se denomina ANOVA o análisis de varianza de un factor, donde cada dimensión es la variable independiente e implica un tratamiento de tamaño $k=4$, que se aplica sobre la muestra elegida.

Los supuestos del diseño son: a) Cada población es independiente; b) La población es el total de profesionales de ingeniería industrial que elige y toma decisiones diversas; c) Tiene distribución normal, donde: $N\left(\mu_{i}, \sigma_{i}^{2}\right)$; d) Las varianzas $\sigma_{i}^{2}$ son iguales a la varianza común $\sigma^{2}$.

Luego de la formulación del constructo, el diseño de la matriz de datos y la elaboración de la herramienta de recolección, se aplicó el Registro de Perfil de Talento Potencial a una muestra estructurada, empleando la técnica de la encuesta para la recolección de información. A continuación se presentan los resultados de la investigación exploratoria realizada a nivel de profesionales de ingeniería Industrial.

\section{Diseño de la muestra}

De acuerdo al objetivo del estudio, se diseño una muestra estructurada. Los participantes se eligieron al azar, dentro de los ciclos VIII a X de los estudiantes de pregrado y de IV ciclo de postgrado. Se consideró un número de treinta y un encuestados, por cada submuestra. Después del diseño de la herramienta de registro de datos, se empleó la técnica de la encuesta para la recolección de información, la cual registra y permite primero, medir los resultados individuales de cada variable por cada una de las dimensiones, segundo, la sistematización y análisis estadístico.

\section{Conceptos estadísticos}

Unidad de análisis: Decisor (alumno) que elige según las dimensiones.

Variable independiente: El factor es cada dimensión (D), que se considera un tratamiento que influye en las predisposiciones de una población. Se asigna los números $1,2,3,4$ a cada $D$.

Existen k tratamientos, donde $\mathrm{k}=4$.

Variable dependiente: La variable aleatoria dependiente es $X$. Es el peso de la elección por cada variable circunstancial, asociada a cada D.

Dato: Calificación asignada a cada elección.

Instrumento de medición: Para cada D (1, 2, 3, 4), se registra los datos de elección de cada alumno.

Población: La población es el total de decisores (alumnos) que realizan elecciones.

Muestra: Se extrae de cada población. El Cuadro 1 presenta la muestra seleccionada.

La muestra es de tamaño $n=31$.

Selección de muestra: Aleatoria (al azar).

\section{Técnica de recolección de datos}

Por cada tratamiento $D$ se consideró un alumno que realiza elecciones.

De cada población $X_{\mathrm{i}} \mathrm{i}=1,2, \ldots \mathrm{k}, \mathrm{k}=4$, se toma una muestra aleatoria de tamaño simple.

Los elementos de la muestra $X_{\mathrm{ij},} \mathrm{j}=1,2, \ldots \mathrm{n}, \mathrm{n}=$ 31 , son variables aleatorias cuya distribución es de la forma: $N\left(\mu_{\mathrm{i}}, \sigma_{\mathrm{i}}^{2}\right)$

4 Talento es la predisposición a aplicar un enfoque metodológico para la resolución de problemas. 
Definición de la muestra

Cuadro 1. Muestra Ingeniería Industrial.

\begin{tabular}{|c|c|c|c|c|}
\hline UNMSM & subtotal & total & $\%$ & \multirow{6}{*}{$\begin{array}{l}\text { Muestra A1 para estudio de talento } \\
\text { potencial }\end{array}$} \\
\hline \multicolumn{4}{|l|}{ Pregrado FII Muestra A1 } & \\
\hline - PPCO & 6 & & & \\
\hline - Mercadotecnia & 7 & & & \\
\hline - Proyectos & 10 & & & \\
\hline - Biblioteca & 8 & 31 & $33.33 \%$ & \\
\hline \multicolumn{4}{|l|}{ UF } & \multirow{8}{*}{$\begin{array}{l}\text { Muestras para control y comparación } \\
\text { con muestra A1 }\end{array}$} \\
\hline Pregrado FII-Muestra A2 & & & & \\
\hline - Ing.Mantenimiento & 21 & & & \\
\hline - Ética Profesional & 10 & 31 & $33.33 \%$ & \\
\hline \multicolumn{4}{|l|}{ Postgrado II-Muestras B1 } & \\
\hline - Ing.Proc.Empresariales & 21 & & & \\
\hline - Logística Internacional & 10 & 31 & $33.33 \%$ & \\
\hline Total & & 93 & $100 \%$ & \\
\hline
\end{tabular}

Fuente: Elaboración propia

\section{Modelo de relación lineal y prueba de hipótesis}

El modelo lineal es un modelo de un factor completamente aleatorio:

$$
x_{i j}=\mu_{i}+\alpha_{i}+\varepsilon_{i j}
$$

Considerando:

$\mu=$ media total

Error o residuo: variables aleatorias que se desvían de la media,

independientes y normales $N\left(0, \sigma_{i}^{2}\right)$

$$
\varepsilon_{\mathrm{ij}}=X_{\mathrm{ij}}-\mu_{\mathrm{i}}
$$

entonces: $X_{i j}=\mu_{i}+\varepsilon_{i j}, i=1,2,3,4, j=1,2, \ldots 31$

\section{Efecto del tratamiento i:}

$$
\alpha_{i}=\mu_{i}-\mu
$$

donde:

$$
\sum_{i=1}^{k} \alpha_{i}=0
$$

\section{1. ${ }^{\circ)}$ Hipótesis}

$$
\mathrm{H}_{0}: \mu_{1}=\mu_{2}=\mu_{3}=\mu_{4}=\mu
$$

Todas las $\mu_{\mathrm{i}}$ son iguales; donde $\mathrm{i}=1,2, . . \mathrm{k}, \mathrm{k}=4$

ó: no hay efecto de $\mathrm{k}$ tratamientos

$H_{1}$ : No todas las $\mu_{i}$ son iguales; donde $j=1,2,3,4$ ó:

Donde:

$\mu_{\mathrm{i}}$ : Media de las elecciones por el tratamiento i

$\left.2^{\circ}\right)$ n.s. y n.c.

Nivel de significancia $\alpha=0.05$

Nivel de confianza $1-\alpha=0.95$

$3^{\circ}$ ) Estadística de prueba:

$$
F=\frac{{ }_{C M T R}}{\mathrm{CME}} \longrightarrow \mathrm{F}_{\alpha} ;(\mathrm{k}-1, \mathrm{n}-\mathrm{k})
$$

Donde:

$$
\begin{aligned}
& \text { CMTR }=\frac{\text { SCTR }}{\mathrm{k}-1} ; \quad \mathrm{k}=4 \\
& \text { CME }=\frac{\mathrm{SCE}}{\mathrm{n}-\mathrm{k}} ; \mathrm{n}=124
\end{aligned}
$$

SCTR = Suma de cuadrados de los tratamientos CMTR = Cuadrados medios de los tratamientos SCE = Suma de cuadrados del error $\mathrm{CME}=$ Cuadrados medios del error 


\section{Se calcula F:}

4..$^{\circ}$ Cálculo mediante SPSS: ver el Cuadro 2, cuadro 3 y Cuadro 4.

Cuadro 2. Factores inter-sujetos

\begin{tabular}{|c|c|c|}
\hline \multicolumn{2}{|l|}{} & N \\
\hline Dimensión & 1 & 31 \\
\hline & 2 & 31 \\
\hline & 3 & 31 \\
\hline & 4 & 31 \\
\hline
\end{tabular}

Cuadro 3. Estadísticos descriptivos Variable dependiente: elección personal

\begin{tabular}{|c|c|c|c|}
\hline Dimensión & Media & Desv. típ. & $\mathbf{N}$ \\
\hline 1 & 25.90 & 3.986 & 31 \\
\hline 2 & 25.00 & 3.183 & 31 \\
\hline 3 & 23.19 & 3.894 & 31 \\
\hline 4 & 25.90 & 4.245 & 31 \\
\hline Total & 25.00 & 3.959 & 124 \\
\hline
\end{tabular}

Cuadro 4. Pruebas de los efectos inter-sujetos - UNMSM pregrado - FII Variable dependiente: elección personal

\begin{tabular}{|l|c|c|c|c|c|}
\hline \multicolumn{1}{|c|}{ Fuente } & $\begin{array}{c}\text { Suma de cuadrados } \\
\text { tipo III }\end{array}$ & gl & $\begin{array}{c}\text { Media } \\
\text { cuadrática }\end{array}$ & F & Significación \\
\hline Modelo corregido & $151.742(\mathrm{a})$ & 3 & 50.581 & 3.417 & .020 \\
\hline Intersección & 77500.000 & 1 & 77500.000 & 5235.726 & .000 \\
\hline DIMENSIÓN & 151.742 & 3 & 50.581 & 3.417 & .020 \\
\hline Error & 1776.258 & 120 & 14.802 & & \\
\hline Total & 79428.000 & 124 & & & \\
\hline Total corregida & 1928.000 & 123 & & & \\
\hline
\end{tabular}

a $\mathrm{R}$ cuadrado $=.079(\mathrm{R}$ cuadrado corregida $=.056)$

Fuente: Acevedo (2009).

\section{5. $\left.{ }^{\circ}\right)$ Prueba $F$ para análisis de varianza de un} factor: En el Gráfico 3.

Gráfico 3. Región de aceptación y rechazo en curva F.

$\mathrm{F}_{\mathrm{\alpha} ;(\mathrm{k}-1, \mathrm{n}-\mathrm{k})}=\mathrm{F}_{0.05 ;(4-1,124-4)}=\mathrm{F}_{0.05 ;(3,120)}=1.96$

Fuente: Acevedo (2009). 
Para las muestras UF pregrado y postgrado, se tiene los siguientes resultados de ANOVA.

Cuadro 5. Pruebas de los efectos inter-sujetos - UF - pregrado FII Variable dependiente: elección personal

\begin{tabular}{|l|c|c|c|c|c|}
\hline \multicolumn{1}{|c|}{ Fuente } & $\begin{array}{c}\text { Suma de cuadrados } \\
\text { tipo III }\end{array}$ & gl & Media cuadrática & F & Significación \\
\hline Modelo corregido & $329.871(\mathrm{a})$ & 3 & 109.957 & 7.591 & .000 \\
\hline Intersección & 77500.000 & 1 & 77500.000 & 5350.581 & .000 \\
\hline DIMENSIÓN UF & 329.871 & 3 & 109.957 & 7.591 & .000 \\
\hline Error & 1738.129 & 120 & 14.484 & & \\
\hline Total & 79568.000 & 124 & & & \\
\hline Total corregida & 2068.000 & 123 & & & \\
\hline
\end{tabular}

a $\mathrm{R}$ cuadrado $=.160(\mathrm{R}$ cuadrado corregida $=.138)$

Fuente: Ibídem

Cuadro 6. Pruebas de los efectos inter-sujetos - UF - EPG MII Variable dependiente: elección personal

\begin{tabular}{|l|c|c|c|c|c|}
\hline \multicolumn{1}{|c|}{ Fuente } & $\begin{array}{c}\text { Suma de cuadrados } \\
\text { tipo III }\end{array}$ & gl & Media cuadrática & F & Significación \\
\hline Modelo corregido & $544.323(\mathrm{a})$ & 3 & 181.441 & 17.014 & .000 \\
\hline Intersección & 77500.000 & 1 & 77500.000 & 7267.457 & .000 \\
\hline DIMENSIÓN EPG & 544.323 & 3 & 181.441 & 17.014 & .000 \\
\hline Error & 1279.677 & 120 & 10.664 & & \\
\hline Total & 79324.000 & 124 & & & \\
\hline Total corregida & 1824.000 & 123 & & & \\
\hline
\end{tabular}

a $\mathrm{R}$ cuadrado $=.298(\mathrm{R}$ cuadrado corregida $=.281)$ Fuente: Ibidem

Cuadro 7. Pruebas de los efectos inter-sujetos total muestras Variable dependiente: elección personal

\begin{tabular}{|l|c|c|c|c|c|}
\hline \multicolumn{1}{|c|}{ Fuente } & $\begin{array}{c}\text { Suma de cuadrados } \\
\text { tipo III }\end{array}$ & gl & Media cuadrática & F & Significación \\
\hline Modelo corregido & $895.505(\mathrm{a})$ & 3 & 298.502 & 22.307 & .000 \\
\hline Intersección & 232500.000 & 1 & 232500.000 & 17374.372 & .000 \\
\hline DIMENSION TOT & 895.505 & 3 & 298.502 & 22.307 & .000 \\
\hline Error & 4924.495 & 368 & 13.382 & & \\
\hline Total & 238320.000 & 372 & & & \\
\hline Total corregida & 5820.000 & 371 & & & \\
\hline
\end{tabular}

a $\mathrm{R}$ cuadrado $=.154(\mathrm{R}$ cuadrado corregida $=. \mathrm{Ibídem}$ propia con SPSS 


\section{$6^{\circ}{ }^{\circ}$ Análisis y decisión en muestra: UNMSM- pregrado FII}

Para un nivel de significación de 0.05 el $\mathrm{F}$ calculado es superior al $\mathrm{F}$ teórico.

Por lo tanto se rechaza la hipótesis nula.

El valor $p=0.020$ indica que existe un $2.0 \%$ de posibilidad de observar diferencias en las medias de elecciones personales en las cuatro dimensiones 1, 2, 3, 4 (alternativas de elección).

\section{Conclusiones de la investigación}

Según los resultados de la prueba de hipótesis, a un nivel de significación de 0.05 , no es posible aceptar la hipótesis de que las medias de las cuatro dimensiones son iguales.

Alternativamente, no se puede rechazar la hipótesis que afirma que las medias de la población no son todas iguales.

- Se rechaza la Hipótesis nula a un nivel de significación de 0.05 .

- Se acepta Hipótesis alternativa un nivel de significación de 0.05 .

\section{RESULTADOS DEL ESTUDIO}

\section{Resultados generales}

Si las medias de las dimensiones no son iguales, entonces, no se puede rechazar la sentencia que afirma que los ingenieros industriales tienen diferente predisposición hacia las dimensiones y poseen diversos perfiles de orientación a la metodología de decisiones. El Gráfico 4 (que no se encuentra a escala) presenta el resultado de la muestra A1, lo que ha permitido identificar la composición de los cuatro perfiles. Como se identificó en el Cuadro 1 , la muestra A1 es de profesionales jóvenes (25-30 años de edad) con poca o ninguna experiencia de trabajo.

El profesional del campo 1 se encuentra en el $13 \%$ de la muestra, es innovador, pragmático, capaz de crear productos que derivan en invenciones y patentes, es el inventor emprendedor que emplea la intuición, creatividad e imaginación, para realizar tareas nuevas y proyectos que aperturan nuevos caminos en la resolución de problemas. Se orienta al empleo de nuevas técnicas y sistemas de actividad humana, que pueden consolidarse como negocios o sectores inéditos. El profesional del campo 2, orientado a los hechos percibibles por los sentidos y las experiencias del mundo real, se encuentra en el $45 \%$ de la muestra, tiende a ser un profesional que enfatiza el análisis y reductibilidad de las situaciones problemáticas, con fines de mejora de la vida y la satisfacción de las preferencias y necesidades. En este campo, los profesionales tienden a enfocarse en actividades que les permiten resolver situaciones problemáticas concretas. El profesional del campo 3 se encuentra en el $19 \%$ de la muestra, es un profesional orientado a la aplicación de teorías para la resolución de problemas, es un diseñador y modelador de sistemas físicos y conceptuales, que tiene un foco pragmático a través de la aplicación de técnicas y herramientas enfocadas en manejar variables controlables para lograr soluciones funcionales. Las carreras técnicas (ingeniería, arquitectura, biología) enfatizan la aplicación de sus teorías para las tareas concretas de resolver problemas en las organizaciones. El profesional del campo 4 se encuentra en el $23 \%$ de la muestra, está orientado a las actividades académicas y sistemáticas, tiende a concentrarse en tareas de investigación y frecuentemente se embarca en proyectos experimentales, los que se orientan a este campo encuentran mayor satisfacción en el manejo de conceptos antes que resolver situaciones del mundo real, su trabajo es ordenado, con metas predefinidas con resultados a largo plazo. 
Gráfico 4. Resultados del estudio muestra A1 (rechazo $\mathrm{H}_{\mathrm{o}}$, no rechazo $\mathrm{H}_{1}$ )

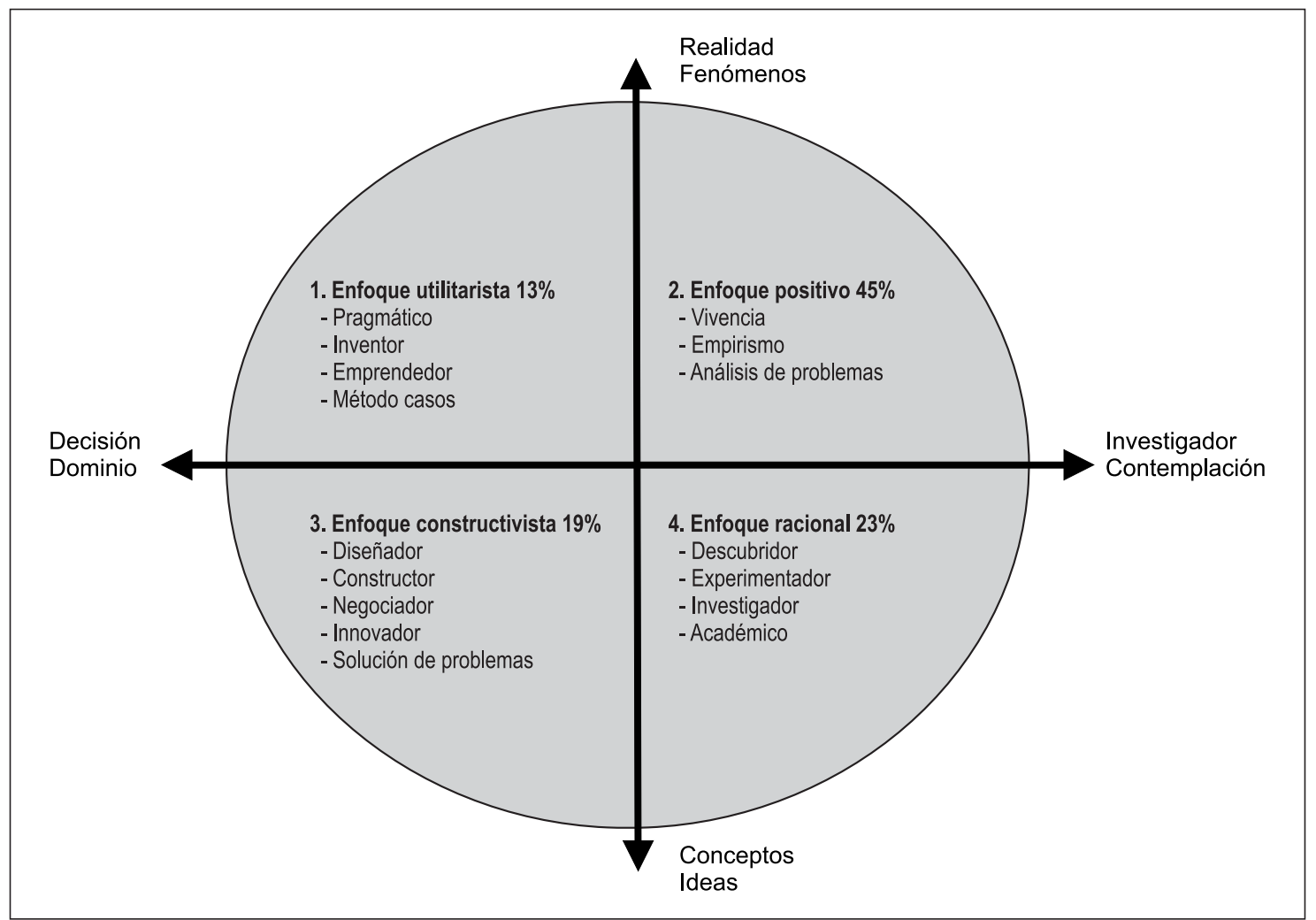

Fuente: Acevedo, Adolfo Ibídem.

\section{Comparación de muestras diferentes}

Comparando las muestras de poblaciones diferentes A2 (semejante a la muestra A1) y la muestra B (profesionales hasta 50 años, con experiencia laboral en niveles directivos). La muestra $\mathrm{A} 1$ se dirige al campo de la vivencia empírica en un $45 \%$, mientras la muestra $\mathrm{A} 2$ y $\mathrm{B}$ se orientan al campo de la experimentación en $39 \%$ y $61 \%$ respectivamente. Es de destacar que el campo 3, relacionado al estudio de las ingenierías no es preponderante en ninguno de los tres casos, aunque en la muestra A1 llega al $19 \%$, superior a los demás (ver Gráfico 5 ).

En todos los casos, las muestras se orientan a la dimensión de la observación contemplativa, lo que permite suponer que existe reducida capacidad o expertise en decisiones y resolución de problemas. A nivel de profesionales jóvenes, no se observa diferencias relevantes en la orientación hacia los hechos concretos o hacia los conceptos y teorías, por otro lado, los profesionales con experiencia se orientan a la conceptualizacón y teorización, definiendo una práctica laboral más cercana al estudio académico que a la resolución de problemas.

\section{CONCLUSIONES}

El estudio, según la validación de hipótesis y los datos estadísticos obtenidos de las muestras aleatorias, ha permitido obtener las siguientes conclusiones:

- Según la hipótesis no rechazada, los profesionales tienen diferente predisposición hacia las dimensiones del conocimiento, lo que conlleva diferente perfil personal para el estudio académico o para las decisiones y resolución de problemas en empresa.

- Según el perfil predominante, los profesionales están más orientados al análisis y estudio, y menos a resolver problemas.

- La orientación al dominio es baja, lo que permite suponer que la formación universitaria no incentiva las habilidades para manejar recursos o personas, o sea, la gerencia de organizaciones.

Dado que los resultados de la presente investigación son a nivel exploratorio, se requiere verificar las dimensiones y variables del modelo conceptual a fin de diseñar una herramienta de gestión que 
permita la asesoría a individuos, grupos, organizaciones y proyectos en los aspectos de definición de capacidades y aptitudes para la toma de decisiones.

A nivel académico, el estudio brinda un conocimiento mayor de las fortalezas y debilidades de la currícula profesional, lo que permitirá replantear los esquemas de desarrollo de conocimientos, aptitudes y competencias, y redefinir temas de elección de carrera, desarrollo profesional, ajuste de perfil competitivo, de manera que los futuros profesiona- les reciban herramientas para el direccionamiento de su curva de aprendizaje y orientación para crear fuentes de competitividad personal.

Aparentemente, los resultados plantean una potencial interrogante de investigación: ¿La currícula de Ingeniería Industrial de las universidades, permite reorientar las predisposiciones o no tiene ninguna influencia en el desarrollo del conocimiento profesional y personal del alumno? Cuya respuesta ha de contribuir a consolidar el producto e imagen de la Universidad, la Facultad, el alumno y el graduado, en el mercado laboral.

Gráfico 5. Comparación de poblaciones: A (profesionales jóvenes) y B (directivos con experiencia).

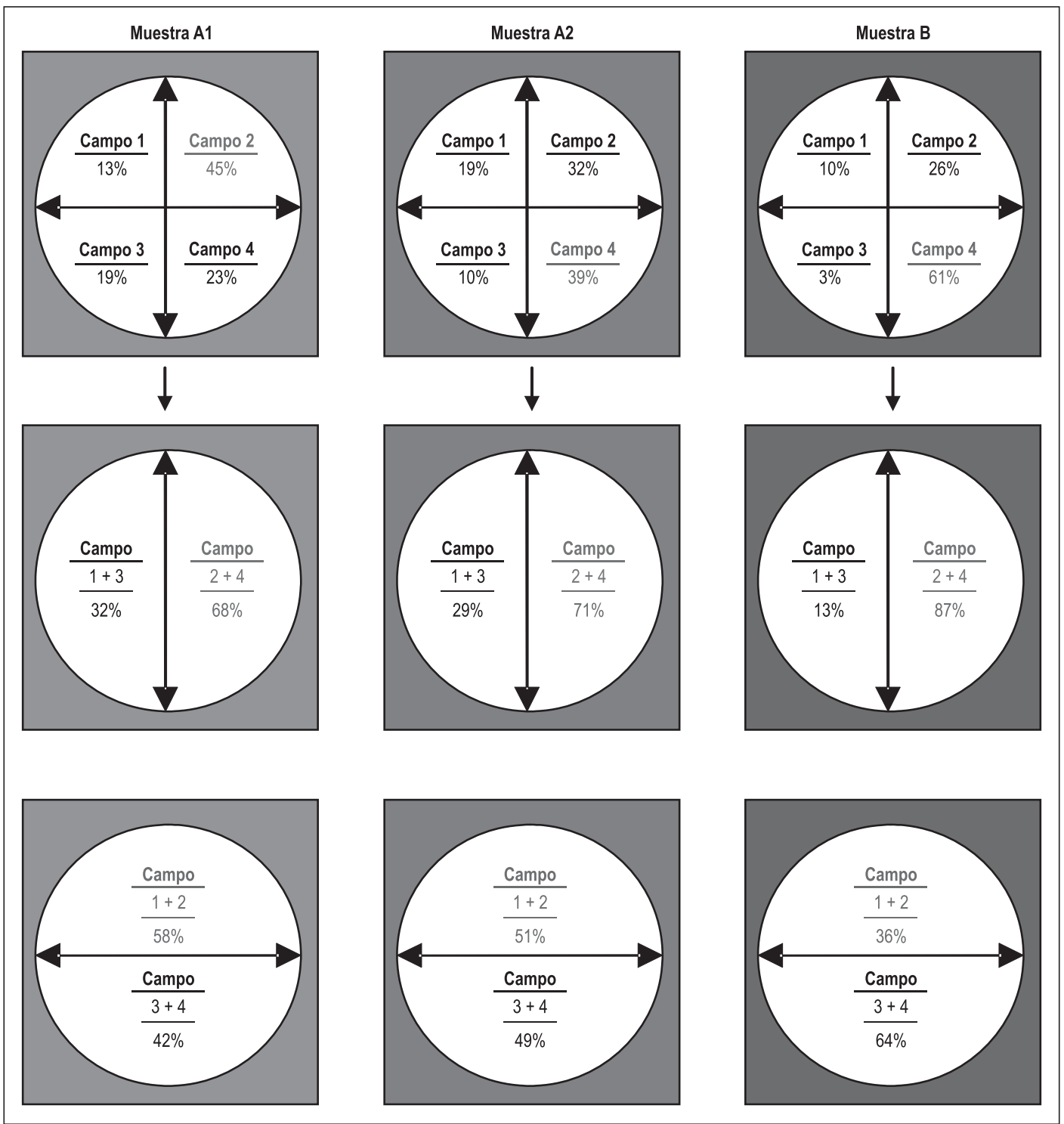

Fuente: Adaptado de Acevedo, Adolfo. Ibidem. 


\section{REFERENCIAS BIBLIOGRÁFICAS}

1. Acevedo, Adolfo (2009). Identificación del talento potencial en los alumnos de Ingeniería Industrial. Informe Técnico FII-UNMSM.

2. Aristóteles (1998). Ética a Nicómaco. Madrid: Editorial ALBA.

3. Blair \& Whitson (1973). Elementos de Ingeniería de Sistemas Industriales. Madrid: Editorial Prentice-Hall Internacional.

4. Carrión, Rolando (2008). "Ingeniería Industrial y Desarrollo". En Industrial Data Vol.11, N. ${ }^{\circ} 1$, enero-junio.

5. Cevallos, Juan (2003). "Hacia una Universidad Nueva”. En Industrial Data Vol. 6, N. ${ }^{\circ} 1$, agosto.

6. Drucker, Peter (1995). El Ejecutivo Eficaz. Editorial: Sudamericana.

7. García, Teonila (2003). "Estimulación de la Creatividad en la Facultad de Ingeniería Industrial para el Desarrollo y la Producción de Software". En Industrial Data Vol. 6, N. 1, agosto.

8. García, Vergiu, Párraga y Santos (2007). “Desarrollo de un modelo Multifactorial y Dinámico para la medición de los intangibles de empresas de manufactura". En Industrial Data, Vol.10 N. ${ }^{\circ} 1$, enero-junio.

9. Hinnells, John (1995). Dictionary of Religions. London: Penguin Books.
10. Hume, David (2005). Investigación sobre el Conocimiento Humano. 2da. edición. Madrid: Mestas Ediciones Escolares.

11. Kolb, Rubin y Maclntyre (1977). Psicología de las Organizaciones: Problemas contemporáneos. Madrid: Prentice/ Hall Internacional.

12. Kuhn, Thomas (1971). La Estructura de las Revoluciones Científicas. México D.F.: Fondo de cultura Económica.

13. Loli, Alejandro (2007). "Compromiso organizacional de los trabajadores de una Universidad Pública" en Industrial Data, Vol.10, N. ${ }^{\circ} 2$, juliodiciembre.

14. McGregor, Douglas (1970). El aspecto humano de la empresa. México: Ed. Diana.

15. Morín, Edgar (1999). Los siete saberes necesarios para la educación del futuro. París. UNESCO.

16. Platón (2003). Diálogos. Obra completa. Madrid: Editorial Gredos.

17. Senge, Peter (1994). La Quinta disciplina en la práctica. Madrid: Granica.

18. Vaill, Peter (1967). "La Ingeniería Industrial y los Sistemas Sociotécnicos". En Sistemas de Producción. Editado por Groff y Muth. Buenos Aires: Ed. El Ateneo. 